\title{
The sensitivity equation method in fluid mechanics
}

\author{
Dominique Pelletier* — Alexander Hay* — Stéphane Etienne* \\ Jeff Borggaard**
}

\author{
* Ecole Polytechnique de Montréal C.P. 6079, Succursalle Centre-Ville \\ Montréal, QC, Canada, H3C $3 A 7$ \\ \{Dominique.Pelletier, Alexander.Hay, Stephanne.Etienne\}@Polymtl.ca \\ ** ICAM Virginia Tech, Blacksburg, VA 24061 \\ jborggaard@vt.edu
}

\begin{abstract}
We present the sensitivity Equation Method (SEM) as a complementary tool to adjoint based optimisation methods. Flow sensitivities exist independently of a design problem and can be used in several non-optimization ways: characterization of complex flows, fast evaluation of flows on nearby geometries, and input data uncertainties cascade through the CFD code to yield uncertainty estimates of the flow field. The Navier-Stokes and sensitivity equationssensitivity are solved by an adaptive finite element method.

RÉSUMÉ. On présente la méthode de l'équation des sensibilités (MES) comme un outil complémentaire aux techniques d'optimisation par variables adjointes. Les sensibilités existent indépendamment du problème d'optimisation et peuvent servir à d'autres fins que le design: caractérisation d'écoulements, évaluation rapide d'écoulements sur des géométries voisines et cascade des incertitudes sur les inputs au travers du code CFD pour obtenir des estimations de l'incertitude sur de l'écoulement. Les équations de Navier-Stokes et des sensibilités sont résolues par une méthode d'éléments finis adaptative.

KEYWORDS: flow sensitivities, Navier-Stokes, optimal design, nearby flows, uncertainty analysis.

MOTS-CLÉS : Sensibilités d'écoulement, Navier-Stokes, conception optimale de forme, solutions voisines, analyse d'incertitude.
\end{abstract}

DOI:10.3166/REMN.17.31-61@ 2008 Lavoisier, Paris

REMN - 17/2008. Shape design in aerodynamics, pages 31 to 61 


\section{Introduction}

A design engineer using CFD must find answers to several questions:

- are the flow predictions obtained with CFD accurate enough for design purposes?

- what are the consequences of changing the parameters controlling the system (boundary conditions, shape parameters etc.)?

- how robust is the design?

- what happens to the flow response if the design or operating parameters suffer from uncertainty?

This paper presents a review of the Sensitivity Equation Method (SEM) as a means of answering the later three questions. The first issue is best dealt with by systematic time-step and grid refinement studies in a numerical analysis process called verification (Roache, 1998) which proceeds in two steps: code verification followed by simulation verification. Code verification ensures, through grid refinement studies, that the discretization algorithm delivers the theoretical rate of convergence on problems with a closed form solution. Simulation verification entails grid error estimation and grid refinement studies to assess a simulation's accuracy and its grid convergence. Whenever possible verification is followed by validation which assess the physical suitability of the mathematical model by comparing verified predictions to quality measurements. See (Roache, 1998) for a thorough discussion. See also (Pelletier et al., 2006; Turgeon et al., 2002) for a discussion of verification issues with adaptive solvers

The word sensitivities has two different meanings. In optimal design it refers to the gradient of the cost function while in fluid mechanics it represents the derivatives of the flow solution (velocity, pressure, temperature etc.) with respect to the parameters of interest. For the flow around an airfoil, $\frac{\partial u}{\partial \alpha}$ is the sensitivity of the velocity with respect to the airfoil angle of attack. It expresses how the velocity field responds to perturbations of $\alpha$ around its nominal value. It is probably better to use the term flow sensitivities. In both cases, however, sensitivities measure the importance of changes in the response (cost function or flow) to perturbations of the design or model parameters.

Flow sensitivities have a an intrinsic meaning while adjoint variables makes sense only when the optimization problem from which they are derived is completely specified. Flow sensitivities find uses that are complementary to that of adjoint variables. They provide information that can be used to produce fast and inexpensive estimates of the flow solution for nearby values of the operating or design parameters without resorting to a full blown flow reanalysis. This is done using Taylor series in parameter space, and is especially useful to answer what if questions for complex flows. Finally, sensitivity information can serve to cascade input data uncertainty through a CFD code to yield uncertainty estimates of the flow response. In both cases speed and cost- 
effectiveness are achieved because sensitivities are obtained at a fraction of the cost of computing the flow.

Sensitivity analysis is a more advanced field in solid mechanics than in fluid dynamics. Indeed, textbooks have been written on sensitivity analysis of structures (Kleiber et al., 1997; Haug et al., 1986). To our knowledge there is only one book on sensitivity analysis of flow problems (Stanley et al., 2001). It is recent and more specialized than structural mechanics books. Also, (Gunzburger, 2002) discusses sensitivity analysis in the context of flow control and optimization.

There are several means of computing flow sensitivities: finite differences of flow solutions, the complex step method (Martins et al., 2003; Lu et al., 2006), automatic differentiation (Putko et al., 2001), and sensitivity equation methods (SEM) (Borggaard et al., 1997; Stanley et al., 2001; Turgeon et al., 2000b). The finite difference approach is a well known technique of estimating derivatives. It is based on the following approximation of the derivative of a function $f$ :

$$
\frac{d f}{d x} \approx \frac{f(x+h)-f(x)}{h}
$$

The truncation error is $O(h)$, and thus this is a first-order approximation of the derivative. Note that in our case, a full Navier-Stokes simulation must be performed for each evaluation of $f$. Higher-order finite difference stencils can be derived, at the cost of additional flow evaluations. This option is thus costly because the problem must be solved for two or more values of each parameter of interest. For example, if $a$ represents a vector of 10 parameters, then 11 flow evaluations are required; one for the baseline values of $\boldsymbol{a}_{0}$, and one per perturbation for each of the 10 parameters. In the case of a shape parameter, further technical problems arise because non matching meshes are obtained for different values of the shape parameter.

The complex-step method as a computational tool for evaluating derivatives was demonstrated by Lyness and Moler (Lyness et al., 1967). It is similar to the finite difference approach and is based on the following properties of complex numbers. Consider a function, $f=u+i v$, of the complex variable, $z=x+i y$. If $\mathrm{f}$ is analytic, then the Cauchy-Riemann equations apply

$$
\begin{aligned}
& \frac{\partial u}{\partial x}=\frac{\partial v}{\partial y} \\
& \frac{\partial u}{\partial y}=\frac{\partial v}{\partial x}
\end{aligned}
$$

The first condition can be used to write the following approximation

$$
\frac{\partial u}{\partial x}=\lim _{h \rightarrow 0} \frac{v(x+i(y+h))-v(x+i y)}{h}
$$


where $\mathrm{h}$ is a real number. We can set $y=0, u(x)=f(x)$ and $v(x)=0$ because the original problem involved only real variables so that equation [5] can then be rewritten as

$$
\frac{\partial f}{\partial x}=\lim _{h \rightarrow 0} \frac{\Im[f(x+i h)]}{h}
$$

For a small discrete $\mathrm{h}$, this can be approximated by

$$
\frac{\partial f}{\partial x} \approx \frac{\Im[f(x+i h)]}{h}
$$

This complex-step derivative approximation is very robust and is not subject to subtractive cancellation errors, since it does not involve a difference operation. However, it requires about twice as much memory as the finite difference approach due to the use of complex variables, and somewhat more CPU time (Anderson et al., 2001).

Automatic differentiation (also known as algorithmic differentiation or computational differentiation) is a well established method for estimating derivatives. The method is based on the application of the chain rule of differentiation to each operation in the program simulating the flow. It is equivalent to differentiating the discrete equations to generate a system of equations for the discrete sensitivities. It is powerful because it automatically generates the code for calculating sensitivities (A.Griewank, 2000). In many cases, implementation requires human intervention to ensure efficiency of the code.

Approaches to calculating sensitivities also differ depending on the order of the operations of approximation and differentiation. In the discrete sensitivity equation approach, the total derivative of the flow approximation with respect to the parameter is calculated (Haug et al., 1986), whereas in the continuous sensitivity equation method (SEM) one differentiates the continuum equations to yield differential equations for the continuous sensitivities (Borggaard et al., 1997). See (Kleiber et al., 1997) for a discussion of the two approaches. We have adopted the latter approach for several reasons. First, in the case of shape parameters, it avoids the delicate issue of computing mesh sensitivities. Second, because the continuous SEM works on the continuum equations, it avoids the issue of differentiating computational facilitators (Gunzburger, 2002) such as upwind schemes in finite volume methods or stabilisation terms in finite element formulations. Finally, the method has shown its robustness in optimisation of noisy cost functions (Borggaard et al., 2002).

Automatic differentiation for first-order flow sensitivities is discussed by (Sherman et al., 1996) and (Putko et al., 2001). Continuous SEMs may be found in (Godfrey et al., 1998; Godfrey et al., 2001; Borggaard et al., 1997; Limache, 2000; Turgeon et al., 2001b) for aerodynamics applications. Application to heat conduction is reported by (Blackwell et al., 1998). Sensitivities for incompressible flows with heat transfer may be found in several references (Borggaard et al., 1998; Turgeon et al., 2000c; Turgeon et al., 2000b; Turgeon et al., 2000a). Sensitivity analysis for turbulence models is detailed in the works by (Godfrey et al., 2001) and by (Turgeon et al., 2001 a; Turgeon 
et al., 2001c). A wide variety of flow regimes were treated by the authors (Turgeon $e t$ al., 1999; Turgeon et al., 2000c; Turgeon et al., 2000b; Turgeon et al., 2000a; Turgeon et al., 2001d; Turgeon et al., 2001c). First and second order SEM were developed for steady and unsteady incompressible flows by the Polytechnique group (Turgeon et al., 2001b; Mahieu et al., 2005; Ilinca et al., 2005; Ilinca et al., 2006).

The paper is organised as follows. Section 2 describes the governing equations and their boundary conditions. This is followed by a derivation of the Sensitivity Equations. The formulation issues for shape parameters are examined in Section 4. Section 5 uses flow over NACA four digit airfoils to discuss numerical issues and grid refinement studies for verification of the implementation in the sense of Roache (Roache, 1998). Sections 6 and 7 present application of the SEM fo fast evaluation of solutions on nearby geometries and a variety of optimal airfoil design problems.

\section{Flow equations}

The flow regime of interest is incompressible and modeled by the Navier-Stokes equations (momentum and continuity equations):

$$
\begin{aligned}
& \tilde{\rho} \frac{\partial \tilde{\boldsymbol{u}}}{\partial \tilde{t}}+\tilde{\rho} \tilde{\boldsymbol{u}} \cdot \tilde{\nabla} \tilde{\boldsymbol{u}}=-\tilde{\nabla} \tilde{p}+\tilde{\boldsymbol{f}}+\tilde{\nabla} \cdot\left[\tilde{\mu}\left(\tilde{\nabla} \tilde{\boldsymbol{u}}+(\tilde{\nabla} \tilde{\boldsymbol{u}})^{T}\right)\right] \\
& \tilde{\nabla} \cdot \tilde{\boldsymbol{u}}=0
\end{aligned}
$$

where $\tilde{\rho}$ is the density, $\tilde{t}$ is time, $\tilde{\boldsymbol{u}}$ is the velocity, $\tilde{p}$ is the pressure, $\tilde{\mu}$ is the viscosity, and $\tilde{\boldsymbol{f}}$ is a body force. The tilde $\sim$ denotes dimensional quantities.

The above system is closed with a proper set of initial conditions

$$
\tilde{\boldsymbol{u}}(\tilde{\boldsymbol{x}}, \tilde{t}=0)=\tilde{\boldsymbol{U}}_{0}(\boldsymbol{x}) \text { in } \Omega
$$

and Dirichlet and Neumann boundary conditions

$$
\begin{aligned}
& \tilde{\boldsymbol{u}}(\tilde{\boldsymbol{x}}, t)=\tilde{\boldsymbol{U}}_{b}(\tilde{\boldsymbol{x}}, \tilde{t}) \text { on } \Gamma_{D} . \\
& \tilde{\boldsymbol{t}}=\left[-\tilde{p} \mathbb{I}+\tilde{\mu}\left(\tilde{\nabla} \tilde{\boldsymbol{u}}+\tilde{\nabla} \tilde{\boldsymbol{u}}^{T}\right)\right] \cdot \hat{\boldsymbol{n}}=\tilde{\boldsymbol{F}}^{N} \text { on } \Gamma_{N}
\end{aligned}
$$

where $\Omega$ is the computational domain, $\tilde{U}_{b}$ is the value of the velocity imposed on the boundary $\Gamma_{D}, \mathbb{I}$ is the identity tensor, and $\tilde{\boldsymbol{F}}^{N}$ is the imposed boundary value of the surface traction force $\tilde{\boldsymbol{t}}$ imposed on the boundary segment $\Gamma_{N}$, and $\hat{\boldsymbol{n}}$ is the outward unit normal to $\Gamma_{N}$. 
We make the equations dimensionless by selecting reference quantities for all variables and physical properties: $\tilde{L}_{0}, \tilde{\rho}_{0}, \tilde{\mu}_{r}, \tilde{U}_{r}, \tilde{p}_{r}=\tilde{\rho}_{r} \tilde{U}_{r}^{2}$ and $\tilde{f}_{r}=\frac{\tilde{\rho}_{r} \tilde{U}_{r}^{2}}{\tilde{L}_{r}}$. This leads to the following dimensionless variables:

$$
\begin{aligned}
& x=\frac{\tilde{x}}{\tilde{L}_{r}}, \quad y=\frac{\tilde{y}}{\tilde{L}_{r}}, \quad u=\frac{\tilde{u}}{\tilde{U}_{r}}, \quad v=\frac{\tilde{v}}{\tilde{U}_{r}}, \\
& p=\frac{\tilde{p}}{\tilde{p}_{r}}, \quad \quad \rho=\frac{\tilde{\rho}}{\tilde{\rho}_{r}}, \quad \mu=\frac{\tilde{\mu}}{\tilde{\mu}_{r}}
\end{aligned}
$$

For constant property flows we have $\rho=1$ and $\mu=1$. Boundary conditions are normalized in the same manner. This leads to the following dimensionless continuity and momentum equations

$$
\begin{aligned}
\rho \frac{\partial u}{\partial t}+\rho \boldsymbol{u} \cdot \nabla \boldsymbol{u} & =-\nabla p+\boldsymbol{f}+\nabla \cdot\left[\frac{\mu}{R e}\left(\nabla \boldsymbol{u}+(\nabla \boldsymbol{u})^{T}\right)\right] \\
\nabla \cdot \boldsymbol{u} & =0
\end{aligned}
$$

with boundary conditions

$$
\boldsymbol{u}(\boldsymbol{x}, t=0)=\boldsymbol{U}_{0}(\boldsymbol{x}) \text { in } \Omega
$$

and Dirichlet and Neumann boundary conditions

$$
\begin{aligned}
\boldsymbol{u}(\boldsymbol{x}, t) & =\boldsymbol{U}_{b}(\boldsymbol{x}, t) \text { on } \Gamma_{D} . \\
\boldsymbol{t} & =\left[-p \mathbb{I}+\mu\left(\nabla \boldsymbol{u}+\nabla \boldsymbol{u}^{T}\right)\right] \cdot \hat{\boldsymbol{n}}=\boldsymbol{F}^{N} \text { on } \Gamma_{N}
\end{aligned}
$$

\section{Sensitivity equations}

\subsection{General formulation of sensitivity equations}

The continuous sensitivity equations (CSE) are derived formally by implicit differentiation of the flow Equations [13] to [14] with respect to an arbitrary model parameter $a$. We treat the variable $\boldsymbol{u}$ as a function of both space and of the parameter $a$. This dependence is denoted as $\boldsymbol{u}(\boldsymbol{x} ; a)$. Defining the flow sensitivities as the partial derivatives $\boldsymbol{s}_{u}=\frac{\partial \boldsymbol{u}}{\partial a}$ and $s_{p}=\frac{\partial p}{\partial a}$, and denoting the derivatives of the fluid properties and other flow parameters by a $\left(^{\prime}\right)$, differentiation of Equations [13] and [14] yields

$$
\begin{aligned}
\rho^{\prime} \frac{\partial \boldsymbol{u}}{\partial t}+\rho \frac{\partial \boldsymbol{s}_{u}}{\partial t}+\rho^{\prime} \boldsymbol{u} \cdot \nabla \boldsymbol{u} & \\
+\rho \boldsymbol{s}_{u} \cdot \nabla \boldsymbol{u}+\rho \boldsymbol{u} \cdot \nabla \boldsymbol{s}_{u}= & -\nabla s_{p}+\boldsymbol{f}^{\prime} \\
+\nabla \cdot & {\left[\mu^{\prime}\left(\nabla \boldsymbol{u}+(\nabla \boldsymbol{u})^{T}\right)\right.} \\
& \left.+\mu\left(\nabla \boldsymbol{s}_{u}+\left(\nabla \boldsymbol{s}_{u}\right)^{T}\right)\right]
\end{aligned}
$$




\subsection{Initial and boundary conditions}

Initial conditions for the sensitivity equations are obtained by implicit differentiation of Equations [15]

$$
\boldsymbol{s}_{\boldsymbol{u}}(\boldsymbol{x}, t=0)=\frac{\partial \boldsymbol{U}_{0}}{\partial a}(\boldsymbol{x}) \text { in } \Omega
$$

Dirichlet conditions are obtained in a similar manner. However, one must account for the fact that the boundary segment $\Gamma_{D}$ may now depend on $a$. Thus we write

$$
\boldsymbol{u}\left(x_{b}(a), y_{b}(a) ; a\right)=\boldsymbol{U}_{b}\left(x_{b}(a), y_{b}(a) ; a\right)
$$

so that one must take the total derivative on both sides to obtain a Dirichlet boundary condition for $s_{\boldsymbol{u}}$ :

$$
\boldsymbol{s}_{\boldsymbol{u}}(\boldsymbol{x}, t ; a)=\frac{d \boldsymbol{U}_{b}}{d a}(\boldsymbol{x}, t ; a)-\frac{\partial \boldsymbol{u}}{\partial x} \frac{\partial x_{b}}{\partial a}-\frac{\partial \boldsymbol{u}}{\partial y} \frac{\partial y_{b}}{\partial a} \quad \text { on } \Gamma_{D}
$$

The first term on the right hand side expresses the dependence of $\boldsymbol{U}_{b}$ on $a$, while $\frac{\partial x_{b}}{\partial a}$ and $\frac{\partial y_{b}}{\partial a}$ are the shape sensitivities of the boundary segment. They vanish if $a$ is a value parameter. Note that the flow gradient multiplies $\frac{\partial x_{b}}{\partial a}$ and $\frac{\partial y_{b}}{\partial a}$. Thus accurate boundary conditions for a shape parameter require accu rate evaluation of the flow gradients at the boundary, a challenging task because accuracy of flow derivatives decreases near boundaries. Duvigneau has developed promising techniques for extracting accurate flow gradients at the boundary (Duvigneau et al., 2005a; Duvigneau et al., 2005b).

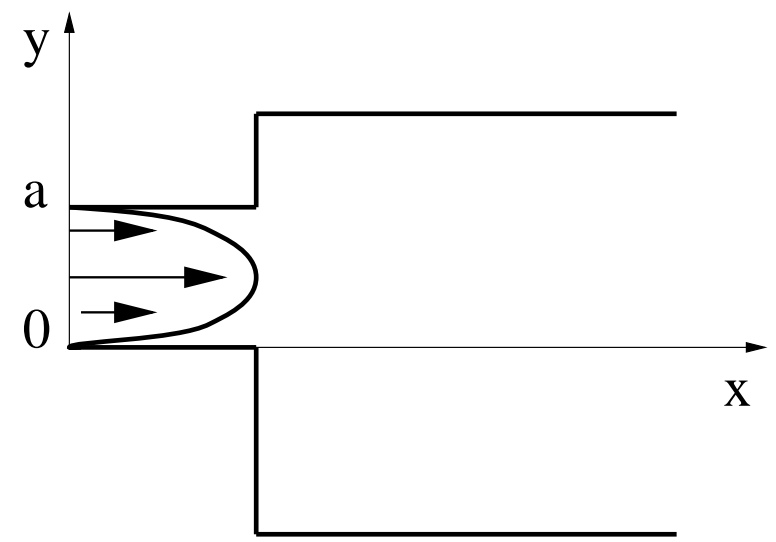

Figure 1. Plenum geometry 
As an example, consider the case of an inlet channel of height $a$ delivering a constant flow rate, $\dot{\mathcal{V}} \mathrm{m}^{2} / \mathrm{s}$ in 2-D, to a plenum independently of the value of $a$ as shown on Figure 1. We have

$$
\begin{aligned}
x_{b} & =0 \\
y_{b} & =a r \\
U_{b} & =\frac{6 r(1-r)}{a} \dot{\mathcal{V}} \quad r \in[0,1]
\end{aligned}
$$

where $r$ is the parametric variable along the boundary and $a$ is the model parameter. For this case the coefficients of Equation [20] are

$$
\begin{aligned}
\frac{\partial x_{b}}{\partial a} & =0 \\
\frac{\partial y_{b}}{\partial a} & =r \\
\frac{d \boldsymbol{U}_{b}}{d a} & =-\frac{6 r(1-r)}{a^{2}} \dot{\mathcal{V}}
\end{aligned}
$$

so that the boundary condition for $s_{u}$ is:

$$
s_{u}=-\frac{6 r(1-r)}{a^{2}} \dot{\mathcal{V}}-\frac{\partial u}{\partial y} r .
$$

If $a$ increases, the channel becomes wider and the inflow velocities decrease accordingly to maintain a constant flow rate at the inlet.

For a value parameter the geometry of the domain does not depend on $a$. Hence, $\frac{\partial x_{b}}{\partial a}$ and $\frac{\partial y_{b}}{\partial a}$ vanish so that the boundary condition reduces to

$$
\boldsymbol{s}_{\boldsymbol{u}}(\boldsymbol{x}, t ; a)=\frac{\partial \boldsymbol{U}_{b}}{\partial a}(\boldsymbol{x}, t ; a) \quad \text { on } \Gamma_{D}
$$

Neumann boundary conditions for the sensitivity of the surface forces $s_{t}=\frac{\partial t}{\partial a}$ given by

$$
\begin{aligned}
\boldsymbol{s}_{\boldsymbol{t}}= & {\left[-s_{p} \mathbb{I}+\mu\left(\nabla \boldsymbol{s}_{\boldsymbol{u}}+\nabla \boldsymbol{s}_{\boldsymbol{u}}^{T}\right)+\mu^{\prime}\left(\nabla \boldsymbol{u}+\nabla \boldsymbol{u}^{T}\right)\right] \cdot \hat{\boldsymbol{n}} } \\
& +\left[-p \mathbb{I}+\mu\left(\nabla \boldsymbol{u}+\nabla \boldsymbol{u}^{T}\right)\right] \cdot \hat{\boldsymbol{n}}^{\prime}
\end{aligned}
$$

are obtained by implicit differentiation of Equation [17]

$$
\boldsymbol{s}_{\boldsymbol{t}}=\frac{d \boldsymbol{F}^{N}}{d a}-\left\{-\nabla p \cdot \boldsymbol{\Pi}_{b} \mathbb{I}+\nabla \mu \cdot \boldsymbol{\Pi}_{b}\left(\nabla \boldsymbol{u}+\nabla \boldsymbol{u}^{T}\right)+\mu \boldsymbol{A}\right\} \cdot \hat{\boldsymbol{n}}
$$

in which

$$
\boldsymbol{\Pi}_{b}=\left(\frac{\partial x_{b}}{\partial a}, \frac{\partial y_{b}}{\partial a}\right)^{T}
$$


and the components of the matrix $\boldsymbol{A}$ are

$$
\begin{aligned}
& a_{11}=2\left\{\frac{\partial^{2} u}{\partial x^{2}} \frac{\partial x_{b}}{\partial a}+\frac{\partial^{2} u}{\partial x \partial y} \frac{\partial y_{b}}{\partial a}\right\} \\
& a_{12}=\left\{\frac{\partial^{2} u}{\partial x \partial y} \frac{\partial x_{b}}{\partial a}+\frac{\partial^{2} u}{\partial y^{2}} \frac{\partial y_{b}}{\partial a}\right\}+\left\{\frac{\partial^{2} v}{\partial x^{2}} \frac{\partial x_{b}}{\partial a}+\frac{\partial^{2} v}{\partial x \partial y} \frac{\partial y_{b}}{\partial a}\right\} \\
& a_{21}=a_{12} \\
& a_{22}=2\left\{\frac{\partial^{2} v}{\partial x \partial y} \frac{\partial x_{b}}{\partial a}+\frac{\partial^{2} v}{\partial y^{2}} \frac{\partial y_{b}}{\partial a}\right\}
\end{aligned}
$$

Note that both of the above conditions require the evaluation of first or second derivatives of the flow at the wall. This constitutes an important challenge for the SEM as numerical differentiation results in reduced accuracy of the boundary conditions and hence, of the sensitivity solution too. Extracting accurate derivatives remains an open research question, although some progress has been made (Duvigneau et al., 2005a; Duvigneau et al., 2005b). In the case of a value parameter, all geometric derivatives vanish and Equation [24] yields the following simplified Neumann conditions:

$$
\boldsymbol{s}_{\boldsymbol{t}}=\frac{\partial \boldsymbol{F}^{N}}{\partial a}(\boldsymbol{x}, t ; a) \quad \text { on } \Gamma_{N}
$$

\section{Formulation issues for shape parameters}

\subsection{Geometrical issues}

As parameter dependent boundaries must be properly described, we focus in this section on some geometrical issues arising with shape parameters before presenting the differentiation of physical quantities. There are two parametrizations that must be treated simultaneously 1 ) the shape of the boundary curve $\Gamma$ is described in terms of the shape parameter $a$ and 2) the position of a point on $\Gamma$ is described in terms of the usual curvilinear coordinate along the curve $\Gamma$. Therefore, any point $\hat{\mathbf{X}}$ of the boundary surface depends both on $a$ and $\xi$ :

$$
\Gamma(a)=\left\{\hat{\mathbf{X}}(\xi, a) \quad \mid \quad \xi \in\left[\xi_{0}, \xi_{1}\right]\right\}
$$

Parameter $a$ determines the shape of $\Gamma$ while $\xi$ determines the location of a point on $\Gamma$.

For this parametrized boundary, the unit normal vectors $\hat{\mathbf{n}}(\xi, a)$ and the unit tangent vector $\hat{\mathbf{t}}(\xi, a)$ can be expressed in terms of the derivatives of $\hat{\mathbf{X}}=[\hat{x} ; \hat{y}]^{T}$ with respect to $\xi$ :

$$
\hat{\mathbf{n}}=\left\|\frac{\partial \hat{\mathbf{X}}}{\partial \xi}\right\|^{-1}\left[-\frac{\partial \hat{y}}{\partial \xi}, \frac{\partial \hat{x}}{\partial \xi}\right]^{T} ; \hat{\mathbf{t}}=\left\|\frac{\partial \hat{\mathbf{X}}}{\partial \xi}\right\|^{-1}\left[\frac{\partial \hat{x}}{\partial \xi}, \frac{\partial \hat{y}}{\partial \xi}\right]^{T}
$$




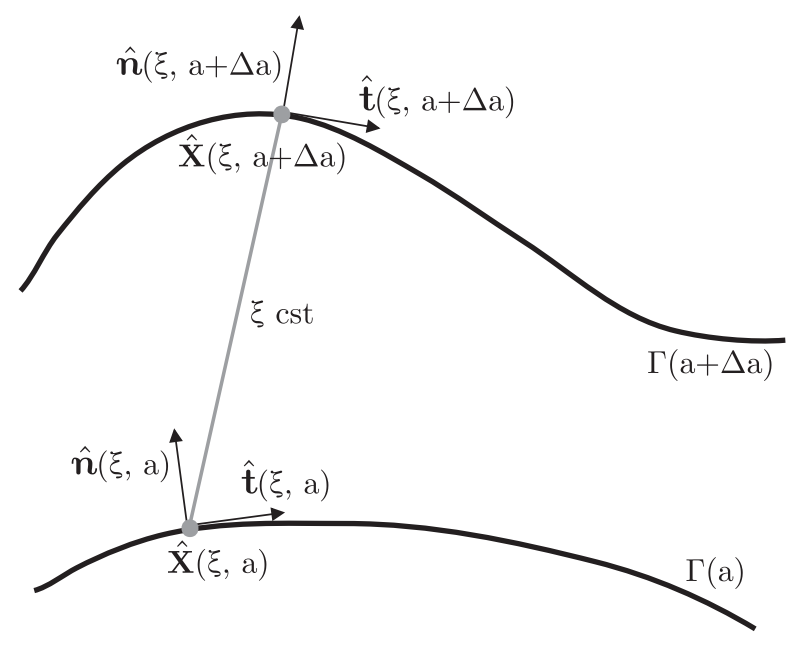

Figure 2. Notations relative to boundary surface

Any change to $a$ will affect all these geometrical quantities. Note that, their material derivatives reduce to their partial derivatives if one uses the curve description Equation [25]. This apparently burdensome notation is key to the successful differentiation of geometrical quantities appearing in sensitivity boundary conditions. If one follows a given point on $\Gamma, \xi$ remains constant as $a$ is varied to change the shape of $\Gamma$ as illustrated in Figure 2. This leads to the following relations:

$$
\begin{aligned}
\frac{D \hat{\mathbf{X}}}{D a}(\xi, a) & =\frac{\partial \hat{\mathbf{X}}}{\partial a}(\xi, a) \\
\frac{D \hat{\mathbf{n}}}{D a}(\xi, a) & =\frac{\partial \hat{\mathbf{n}}}{\partial a}(\xi, a) \\
\frac{D \hat{\mathbf{t}}}{D a}(\xi, a) & =\frac{\partial \hat{\mathbf{t}}}{\partial a}(\xi, a)
\end{aligned}
$$

where $D / D a$ denote the material derivative with respect to $a$. Finally, from Equations [25] and [26], the sensitivities of the unit normal and tangent vectors are derived:

$$
\begin{aligned}
\frac{\partial \hat{\mathbf{n}}}{\partial a}=\left\|\frac{\partial \hat{\mathbf{X}}}{\partial \xi}\right\|^{-1}\left[+\left(\frac{\frac{\partial \hat{\mathbf{X}}}{\partial \xi} \cdot \frac{\partial^{2} \hat{\mathbf{x}}}{\partial \xi \partial a}}{\left\|\frac{\partial \hat{\mathbf{x}}}{\partial \xi}\right\|^{2}}\right) \frac{\partial \hat{y}}{\partial \xi}\right. & -\frac{\partial^{2} \hat{y}}{\partial \xi \partial a}, \\
& \left.-\left(\frac{\frac{\partial \hat{\mathbf{x}}}{\partial \xi} \cdot \frac{\partial^{2} \hat{\mathbf{x}}}{\partial \xi \partial a}}{\left\|\frac{\partial \hat{\mathbf{x}}}{\partial \xi}\right\|^{2}}\right) \frac{\partial \hat{x}}{\partial \xi}+\frac{\partial^{2} \hat{x}}{\partial \xi \partial a}\right]^{T}
\end{aligned}
$$




$$
\begin{aligned}
\frac{\partial \hat{\mathbf{t}}}{\partial a}=\left\|\frac{\partial \hat{\mathbf{X}}}{\partial \xi}\right\|^{-1}\left[-\left(\frac{\frac{\partial \hat{\mathbf{x}}}{\partial \xi} \cdot \frac{\partial^{2} \hat{\mathbf{x}}}{\partial \xi \partial a}}{\left\|\frac{\partial \hat{\mathbf{x}}}{\partial \xi}\right\|^{2}}\right) \frac{\partial \hat{x}}{\partial \xi}\right. & +\frac{\partial^{2} \hat{x}}{\partial \xi \partial a}, \\
& \left.-\left(\frac{\frac{\partial \hat{\mathbf{x}}}{\partial \xi} \cdot \frac{\partial^{2} \hat{\mathbf{x}}}{\partial \xi \partial a}}{\left\|\frac{\partial \hat{\mathbf{x}}}{\partial \xi}\right\|^{2}}\right) \frac{\partial \hat{y}}{\partial \xi}+\frac{\partial^{2} \hat{y}}{\partial \xi \partial a}\right]^{T}
\end{aligned}
$$

\subsection{Evaluating the gradient of a scalar field}

We consider here two local quantities (i.e. the pressure and skin friction coefficients considered at a fixed position) on the parameter dependent boundaries that occur frequently in aerodynamic problems. The following methodology can be applied to any other local quantity.

For incompressible flow, the pressure coefficient is defined by:

$$
C_{p}=\frac{p-p_{\infty}}{\frac{1}{2} \rho U_{\infty}^{2}}
$$

Using the chain rule, the material derivative of the pressure on a boundary surface is given by:

$$
\frac{D p}{D a}=s_{p}+\nabla p \cdot \frac{\partial \hat{\mathbf{X}}}{\partial a}
$$

and thus:

$$
\frac{D C_{p}}{D a}=\frac{1}{\frac{1}{2} \rho U_{\infty}^{2}}\left(s_{p}+\nabla p \cdot \frac{\partial \hat{\mathbf{X}}}{\partial a}\right)
$$

The material derivative includes two separate contributions: the (Eulerian) sensitivity term $s_{p}$ and an additional expression sometimes called the transpiration term in mixed Eulerian/Lagrangian methods $\nabla p \cdot \frac{\partial \hat{\mathbf{X}}}{\partial a}$. The latter ensures that the computed value is correctly located in space. That is, it transports the physical point of interest with the deformation of space due to the change in the value of the shape parameter.

The same approach applies to the skin friction coefficient which is defined as:

$$
C_{f}=\frac{\tau \cdot \hat{\mathbf{n}} \cdot \hat{\mathbf{t}}}{\frac{1}{2} \rho U_{\infty}^{2}}
$$


where $\tau$ is the Cauchy stress tensor and $\tau \cdot \hat{\mathbf{n}} \cdot \hat{\mathbf{t}}$ represents the force acting in the direction tangent to the boundary. In this case, the differentiation is somewhat more involved because the unit normal and tangent vectors to the boundary surface are also parameter dependent. Therefore, the expression for the extrapolated $C_{f}$ involves additional terms describing the effects of $a$ on $\hat{\mathbf{n}}$ and $\hat{\mathbf{t}}$. Keeping in mind that $\hat{\mathbf{n}}$ and $\hat{\mathbf{t}}$ are written as $\hat{\mathbf{n}}(\xi, a)$ and $\hat{\mathbf{t}}(\xi, a)$, their material derivatives with respect to $a$ reduces to their partial derivatives. Thus, we obtain:

$$
\begin{aligned}
\frac{D C_{f}}{D a} & =\frac{1}{\frac{1}{2} \rho U_{\infty}^{2}}\left[\frac{D(\tau \cdot \hat{\mathbf{n}} \cdot \hat{\mathbf{t}})}{D a}\right] \\
& =\frac{1}{\frac{1}{2} \rho U_{\infty}^{2}}\left[\frac{D \tau}{D a} \cdot \hat{\mathbf{n}} \cdot \hat{\mathbf{t}}+\tau \cdot\left(\frac{\partial \hat{\mathbf{n}}}{\partial a} \cdot \hat{\mathbf{t}}+\frac{\partial \hat{\mathbf{t}}}{\partial a} \cdot \hat{\mathbf{n}}\right)\right] \\
& =\frac{1}{\frac{1}{2} \rho U_{\infty}^{2}}\left[\left(s_{\tau}+\nabla \tau \cdot \frac{\partial \hat{\mathbf{X}}}{\partial a}\right) \cdot \hat{\mathbf{n}} \cdot \hat{\mathbf{t}}+\tau \cdot\left(\frac{\partial \hat{\mathbf{n}}}{\partial a} \cdot \hat{\mathbf{t}}+\frac{\partial \hat{\mathbf{t}}}{\partial a} \cdot \hat{\mathbf{n}}\right)\right][29]
\end{aligned}
$$

\subsection{Evaluating the gradient of an integral quantity}

Finally, in optimal design, the objective function measures the performance of the mechanical system of interest. In wing design, the objective function is often built from the aerodynamic forces applied by the fluid to the airfoil. Thus, the objective function involves quantities that are integrated over a parameter dependent surface which take the following generic form:

$$
\int_{\Gamma(a)} \boldsymbol{\Phi} \cdot \hat{\mathbf{n}} d \Gamma
$$

The gradient-based optimization algorithm requires the computation of its derivative with respect to $a$ which is obtained from:

$$
\frac{D}{D a} \int_{\Gamma(a)} \boldsymbol{\Phi} \cdot \hat{\mathbf{n}} d \Gamma=\int_{\Gamma(a)}\left(\frac{D \boldsymbol{\Phi}}{D a} \cdot \hat{\mathbf{n}}+\boldsymbol{\Phi} \cdot \frac{\partial \hat{\mathbf{n}}}{\partial a}+\frac{1}{J} \frac{\partial J}{\partial a} \boldsymbol{\Phi} \cdot \hat{\mathbf{n}}\right) d \Gamma
$$

where $J$ is the Jacobian of the transformation parametrizing the boundary surface as described in Section 4.1 and is given by:

$$
J=\left\|\frac{\partial \hat{\mathbf{X}}}{\partial \xi}\right\|
$$

Its derivative is obtained from:

$$
\frac{\partial J}{\partial a}=\frac{1}{J}\left(\frac{\partial \hat{\mathbf{X}}}{\partial \xi} \cdot \frac{\partial^{2} \hat{\mathbf{X}}}{\partial \xi \partial a}\right)
$$

Finally, $\frac{D \Phi}{D a}$ is calculated from Equation [27], the flow variables, and their sensitivities. 


\section{Application context and numerical framework}

\subsection{Problem statement}

We now apply the proposed method to NACA 4-digit series family of airfoils at a Reynolds number of 1000 . The configuration of the problem is described in Figure 3. Dirichlet boundary conditions are imposed at the inflow and on the airfoil surface, and homogeneous Neumann boundary conditions are prescribed as far field conditions.

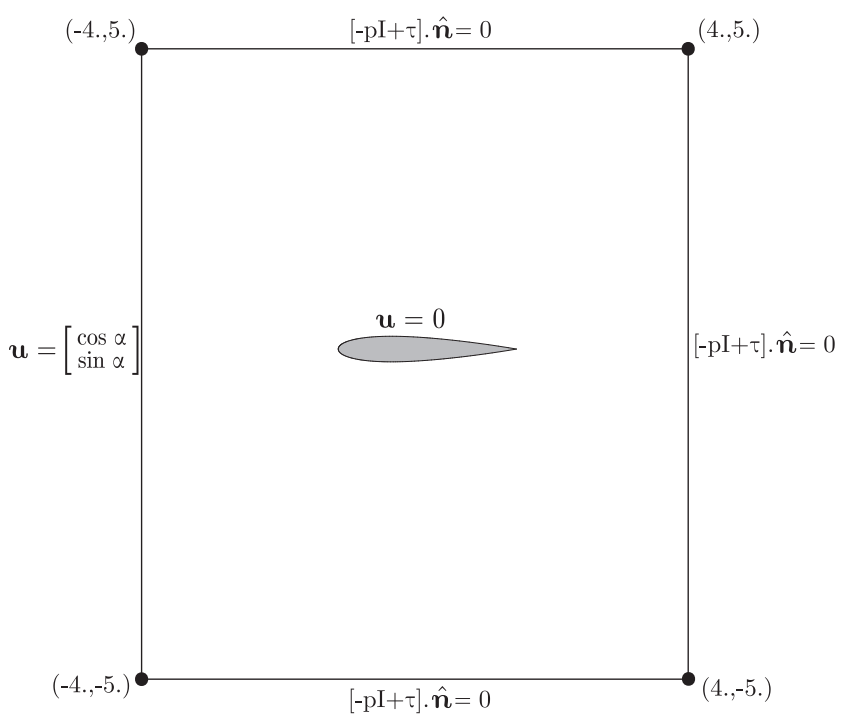

Figure 3. Geometry and boundary conditions for the flows over NACA airfoils

The cambered wing sections of the NACA 4-digit series family of airfoils are obtained by combining a mean line and a thickness distribution. In what follows, we consider unit-chord profiles. The shape of the mean line is expressed analytically by two parabolic arcs tangent at the position of maximum mean-line ordinate. The mean line distribution is given by:

$$
\begin{array}{lll}
y_{m l}= & \frac{m}{p^{2}}\left[2 p x-x^{2}\right] & 0 \leq x \leq x_{p} \\
y_{m l}= & \frac{m}{(1-p)^{2}}\left[(1-2 p)+2 p x-x^{2}\right] & x_{p} \leq x \leq c
\end{array}
$$

where $m$ is the maximum ordinate of the mean line and $p$ is the chord wise position of the maximum ordinate. Both of them are expressed as a fraction of the chord $c$. The thickness distribution is given by the following equation:

$$
y_{t h}= \pm 5 e\left(0.2969 \sqrt{x}-0.1260 x-0.3537 x^{2}+0.2843 x^{3}-0.1015 x^{4}\right)
$$


where $e$ is the maximum thickness of the wing section. Thus, in these equations, the parameters $m$ and $p$ control the camber of the airfoils while the parameter $e$ controls its thickness. All three are shape parameters because they influence the geometry of the domain. The angle of incidence $\alpha$ of the configuration is set by imposing the proper inflow incidence via the inflow Dirichlet boundary condition (see Figure 3). Thus, this parameter is treated as a value parameter.

\subsection{Numerical techniques}

The flow and sensitivity equations are solved by an adaptive finite-element method (Pelletier et al., 1997; Pelletier, 1999). We discuss the salient features here for completeness and to set up the approximation of the continuous sensitivity equation above. The weak form of the continuity and momentum equations are formed, and a discretization based on the 7-noded Crouzeix-Raviart element pair $\left(P_{2}^{+}-P_{1}\right)$ leads to a system of nonlinear algebraic equations which are then solved by Newton's method.

The accuracy of the finite-element approximation is directly related to the local mesh size used. An adaptive remeshing procedure is employed to improve the accuracy, by refining the mesh in regions of high error in the flow and sensitivity variables. Regions targeted for refinement are identified by using an error estimator based on local projections of discontinuous quantities onto a local continuous polynomial basis (Zienkiewicz et al., 1992a; Zienkiewicz et al., 1992b). For example, since the Crouzeix-Raviart element pair uses a piecewise quadratic basis for the velocity, the stress tensor is discontinuous. By projecting it onto a continuous polynomial basis, we can derive an error estimate defined as the difference between the finite-element and the projected stresses.

Once error estimates are obtained for all variables (flow and sensitivities), an optimal mesh is determined using the asymptotic convergence rate of the finite-element method. The optimal mesh is generated to redistribute the mesh sizes so that each element has the same contribution to the norm of the total error:

$$
\|E\|_{\Omega}^{2}=\sum_{k=1}^{n e}\|E\|_{\Omega_{k}}^{2}=n e\left\|E_{e q u i}\right\|^{2}
$$

where the subscripts $\Omega, \Omega_{k}$ refer to norms over the whole domain and the surface of element $k$ respectively. Subscript equi represents the target error obtained by equidistribution of the error over the mesh. Thus when equidistribution is achieved the norm of the error on any element of the mesh has the same value $\left\|E_{\text {equi }}\right\|^{2}$. Finally ne is the number of elements in the mesh. Comparison of the target error to the actual error provides the information needed for refining or coarsening the mesh. The process is performed in an iterative fashion, beginning with a coarse mesh and producing a sequence of meshes which reduce the error by a constant factor over that of the previous mesh usually in the range $[0.25,0.5]$. Details of this adaptive remeshing pro- 
cedure may be found in the literature (Peraire et al., 1987; Pelletier, 1999; Turgeon et al., 1999).

\subsection{Grid convergence study}

In the next Section, the flow around a NACA 4512 airfoil at an incidence of $5^{\circ}$ serves as a baseline reference. The NACA 4512 profile is shown in Figure 4(a), it has a maximum thickness of $12 \%$, a maximum camber displacement of $4 \%$ located at $50 \%$ of chord. $4 \% c$ of maximum ordinate located at the middle of the chord (see Table 1 for the corresponding values of $e, m$ and $p$ ).

Using this configuration, the flow and sensitivity fields are solved using the adaptive finite-element method. All flow and sensitivity variables contribute to the error estimation and mesh adaptation processes. We look at the grid convergence of the aerodynamic forces and their gradients with respect to the shape parameters to assess the influence of the number of grid points on them and to determine practical numerical parameters for the design optimization exercise.

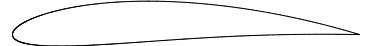

(a) NACA 4512

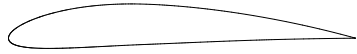

(d) NACA 4412

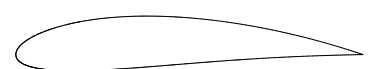

(b) NACA 4515

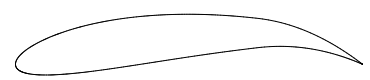

(e) NACA 9714

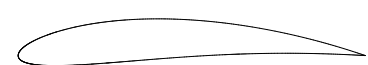

(c) NACA 5512

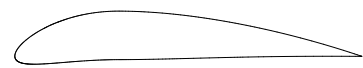

(f) NACA 6314

Figure 4. Geometries of some NACA airfoils

Seven grid adaptation cycles have been performed and the final meshes contain approximately 80000 nodes. Figure 5 shows the last adapted grid in the vicinity of the profile. The evolutions of the Drag coefficient and its derivatives during the adaptive procedure are plotted in Figure 6(a). The derivatives are the material derivatives as computed by Equation [31] with respect to each of the three shape parameters $e, m$ and $p$. Furthermore, the absolute value of the derivatives are considered so that they can be plotted in log-scale. The data on Figure 6(b) shows connvergence for the Lift coefficient.

As can be seen, all the quantities converge to an almost grid independent value with mesh refinement. However, the changes in the gradient values are far larger than those of the aerodynamic coefficients. Indeed, the evaluation of the gradient is more delicate than that of the primal function, since global force sensitivities all depend on the flow sensitivities which, in turn, depend on the flow gradient at the wall through the boundary conditions of the CSE. This study also indicates that the dependence of the Drag (or Lift) and their gradients on the mesh are respectively $1.10^{-5}$ (and $1.10^{-3}$ ). These observations will help us to determine a stopping criterion for the optimization 
procedure. Furthermore, the convergence of these quantities required at least 50000 nodes. As a consequence, in the remainder of this work, six to seven grid adaptation cycles are performed for all reported computations. The reduction factor of the error in the adaptive procedure is set so that the final meshes contain approximately 60000 nodes in all the cases.

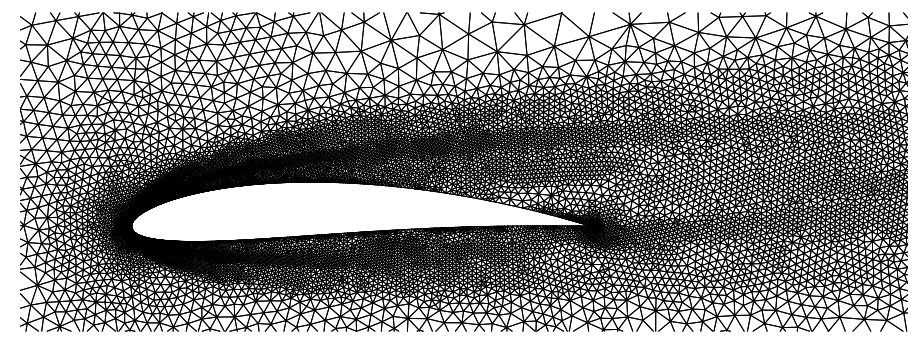

Figure 5. Adapted mesh around the NACA 4512 airfoil ( $R e=1000$ and $\left.\alpha=5^{\circ}\right)$

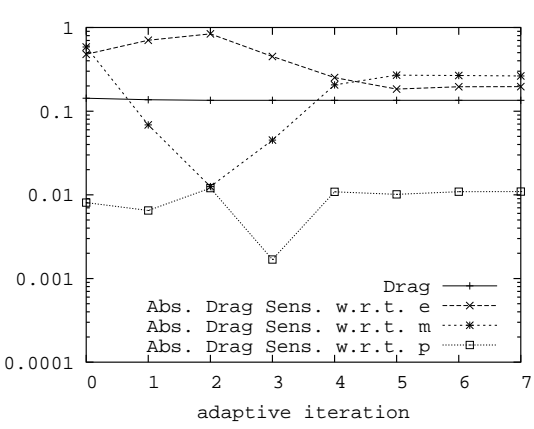

(a) Drag and its derivatives

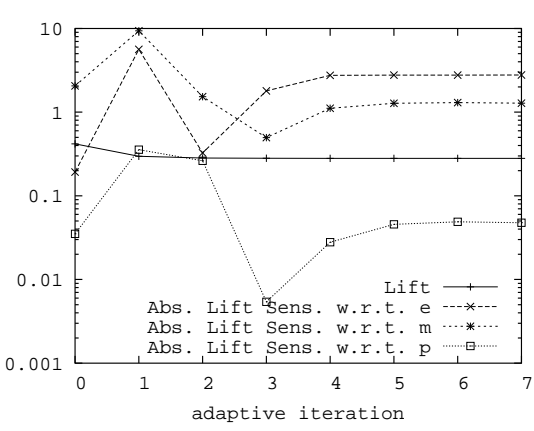

(b) Lift and its derivatives

Figure 6. Mesh refinement study

\section{Application: fast evaluation of flows on nearby geometries}

\subsection{Setting}

This Section focuses on the computations of local and integral quantities on nearby geometries using the CSE method with shape parameters. The main interest of this approach is the capability to estimate outputs on nearby geometries without having to perform a full flow reanalysis. Simplicity and cost-effectiveness are achieved by using Taylor series in parameter space as long as the predicted values are accurate. Such a procedure finds a wide range of useful engineering applications. In the framework of optimization algorithms, it can reduce the cost of both first-order deterministic algorithms and of non-deterministic approaches such as genetic algorithms. Indeed, these 
algorithms require a large number of flow analysis that is responsible for the bulk of the CPU costs. Thus, fast evaluation of flows for nearby values of the parameters can significantly reduces the CPU-time consumed for each optimization exercise.

Let $a_{0}$ denote the baseline value of $a$ and $\Delta a$ its perturbation. Let $\phi$ be any solution variable (say stagnation pressure, $C_{f}, C_{L}$, etc), its value for $a_{0}+\Delta a$ is obtained by linear Taylor series in $a$-space using values of the flow and its sensitivities from the baseline solution. The first order Taylor series extrapolated field is given by:

$$
\phi\left(\hat{\mathbf{X}}, a_{0}+\Delta a\right) \approx \phi\left(\hat{\mathbf{X}}, a_{0}\right)+\Delta a \frac{D \phi}{D a}\left(\hat{\mathbf{X}}, a_{0}\right)
$$

where $\phi$ will be either a local quantity (pressure or skin friction coefficient) or an integral quantity (Drag, Lift and Moment coefficient). The corresponding material derivative is computed as detailed in Section 4. In what follows, $a$ will in turn be $e, m$ and $p$, the shape parameters that define an airfoil of the NACA 4-digit family.

\subsection{Numerical result}

We first perturb each parameter separately. From the baseline airfoil, a $25 \%$ increase in the airfoil thickness parameter $e$ leads to the NACA 4515 airfoil shown in Figure 4(b). The same relative increase in $m$ produces the NACA 5512 airfoil shown in Figure 4(c). And, a 20\% increase in the maximum camber ordinate location $p$ yields the NACA 4412 airfoil shown in Figure 4(d). The corresponding parameters of all these NACA airfoils are reported in Table 1.

Table 1. Parameters of some NACA airfoils (unit chord)

\begin{tabular}{lcccccc}
\hline NACA & 4512 & 4515 & 5512 & 4412 & 9714 & 6314 \\
\hline \hline$e$ & 0.12 & 0.15 & 0.12 & 0.12 & 0.14 & 0.14 \\
\hline$m$ & 0.04 & 0.04 & 0.05 & 0.04 & 0.09 & 0.06 \\
\hline$p$ & 0.50 & 0.50 & 0.50 & 0.40 & 0.70 & 0.30 \\
\hline
\end{tabular}

We now compare the flow solutions obtained by either a full flow reanalysis or by Taylor series extrapolation from the baseline flow. Table 2 reports the values of the aerodynamic coefficients evaluated by the two approaches. As can be seen, even when the percentage changes from the baseline values are substantial, the extrapolated coefficients are in very good agreement with those obtained by reanalysis. In all cases, the relative differences are much less than one percent which confirms the capability of the proposed methodology for computing the material derivatives of integral quantities.

Figures 7,8 and 9 show the pressure coefficient and the skin friction distributions for the baseline flow, the Taylor series estimates and the recomputed flows around the NACA 4515, NACA 5512 and NACA 4412 airfoils. As can be seen, the agreement 
between computations and extrapolations are almost perfect for all the surface coefficients on both the pressure and suction sides of the airfoils. It is worth noting that without the transpiration terms (see Equations [28] and [29]), the predictions are no longer accurate as already reported in (Hay et al., 2006).

Table 2. Extrapolation from a NACA 4512: Aerodynamic coefficients

\begin{tabular}{cccc}
\hline \hline \multirow{2}{*}{ NACA } & Coeff. & $\begin{array}{c}\text { Computed } \\
\text { (\% Change from baseline) }\end{array}$ & $\begin{array}{c}\text { Extrapolated } \\
\text { (\% Diff. with computed) }\end{array}$ \\
\hline \hline \multirow{2}{*}{$\begin{array}{c}4512 \\
\text { (baseline) }\end{array}$} & $C_{D}$ & 0.13474 & \\
\cline { 2 - 4 } \multirow{2}{*}{4515} & $C_{L}$ & 0.28024 & $0.14063(0.3 \%)$ \\
\cline { 2 - 4 } & $C_{D}$ & $0.14106(4.7 \%)$ & $0.19694(0.6 \%)$ \\
\hline \hline \multirow{2}{*}{5512} & $C_{L}$ & $0.19570(30.2 \%)$ & $0.13738(0.1 \%)$ \\
\cline { 2 - 4 } & $C_{D}$ & $0.13756(2.1 \%)$ & $0.29304(0.2 \%)$ \\
\hline \multirow{2}{*}{4412} & $C_{L}$ & $0.29256(4.4 \%)$ & $0.13584(0.1 \%)$ \\
\cline { 2 - 4 } & $C_{D}$ & $0.13591(0.9 \%)$ & $0.14964(1.4 \%)$ \\
\hline \multirow{2}{*}{9714} & $C_{L}$ & $0.27613(1.5 \%)$ & $0.29823(3.5 \%)$ \\
\cline { 2 - 4 } & $C_{D}$ & $0.15180(12.7 \%)$ & $0.14612(1.6 \%)$ \\
\hline \multirow{2}{*}{6314} & $C_{L}$ & $0.30916(10.3 \%)$ & $0.24077(7.8 \%)$ \\
\cline { 2 - 4 } & $C_{D}$ & $0.14853(10.2 \%)$ & $0.2 \%)$ \\
\hline \hline
\end{tabular}

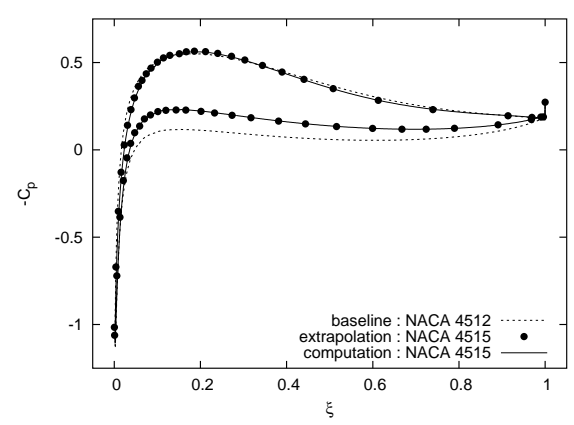

(a) Pressure coefficient

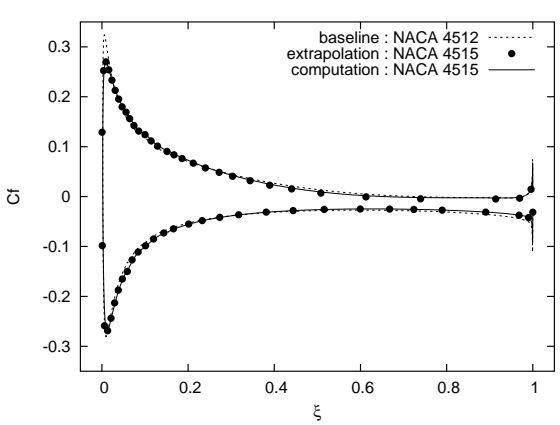

(b) Skin friction coefficient

Figure 7. Nearby solution for the NACA 4515 from the NACA 4512

Now that the procedure has been verified using the previous numerical examples, we seek to evaluate how the linear Taylor series extrapolation performs when facing significant geometrical changes. To this end, we consider the NACA 9714 airfoil shown in Figure 4(e) which differs significantly from the geometry of the NACA 4512 baseline airfoil illustrated in Figure 4(a). All three shape parameters must simultaneously increase, as reported in Table 1, to change the airfoil geometry from that of a 


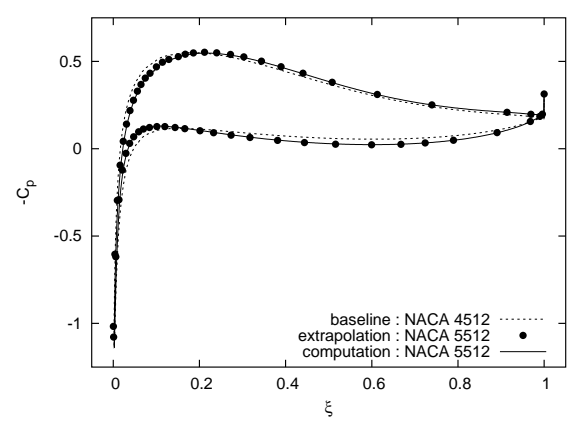

(a) Pressure coefficient

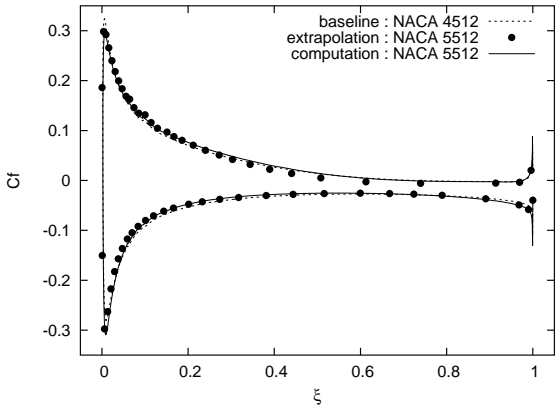

(b) Skin friction coefficient

Figure 8. Nearby solution for the NACA 5512 from the NACA 4512

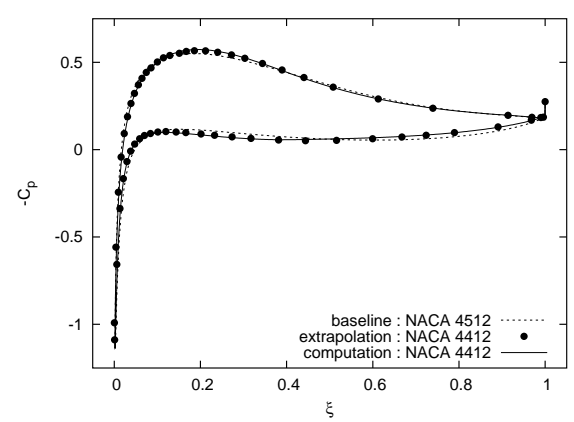

(a) Pressure coefficient

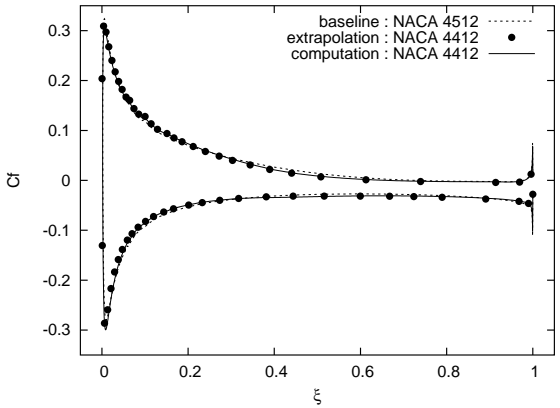

(b) Skin friction coefficient

Figure 9. Nearby solution for the NACA 4412 from the NACA 4512

NACA 4512 to that of a NACA 9714. The relatives changes in $e, m$ and $p$ are respectively $17 \%, 125 \%$ and $40 \%$. Table 2 compares the recomputed and extrapolated aerodynamic coefficients. The discrepancy is more important than for the previous cases, an indication that we are approaching the predictive limits of the first order Taylor series extrapolation for these very demanding geometry changes. However, the predictions are still fairly good considering how large the relative changes are from baseline for these coefficients and thus how important the corresponding geometrical changes are.

Figure 10 shows the comparison between the extrapolated solution and the recomputed solution for both the pressure coefficient and the skin friction. Once again, the discrepancy is more substantial than for the previous cases. The peaks are not fully captured by the extrapolated solution. This holds for the pressure and the skin friction coefficients. The main discrepancy is observed for the pressure coefficient in the 
vicinity of the trailing edge on the pressure side. However, the Taylor series coefficients distributions have captured successfully all the main features in the changes from baseline.

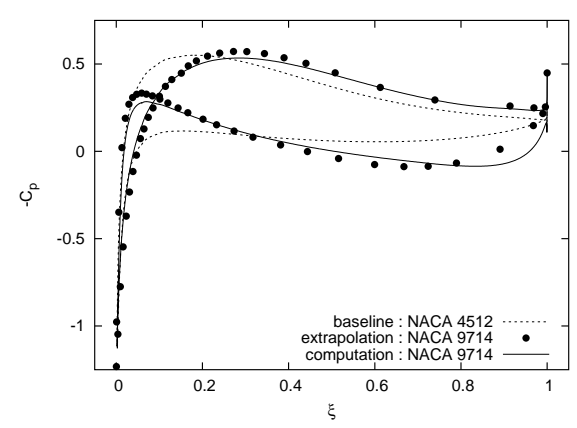

(a) Pressure coefficient

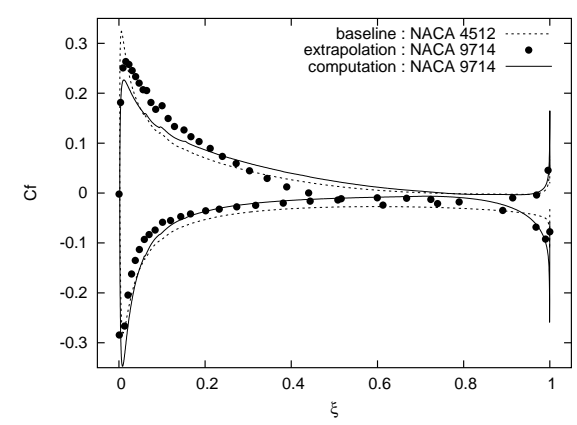

(b) Skin friction coefficient

Figure 10. Nearby solution for the NACA 9714 from the NACA 4512

We complete this Section by obtaining estimates of the flow over a NACA 6314 by extrapolation from the flow over a NACA 4512 . The NACA 6314 is obtained from the NACA 4512 by increasing the thickness by $17 \%$, the maximum camber parameter by $50 \%$ and by decreasing the maximum camber position parameter by $40 \%$ (see Table 1). As can be seen in Table 2, the relative changes in the aerodynamic coefficients is even larger than for the previous case. Yet, the extrapolated Drag coefficient is still in good agreement with the recomputed one. But, the relative difference in the Lift coefficient is larger than $7 \%$ which illustrates the limits of the first order Taylor series extrapolation in terms of the relative changes in a set of parameters. However, as can be seen in Figure 11, the extrapolated local coefficients match the fully recomputed solution fairly well except for the skin friction suction peak.

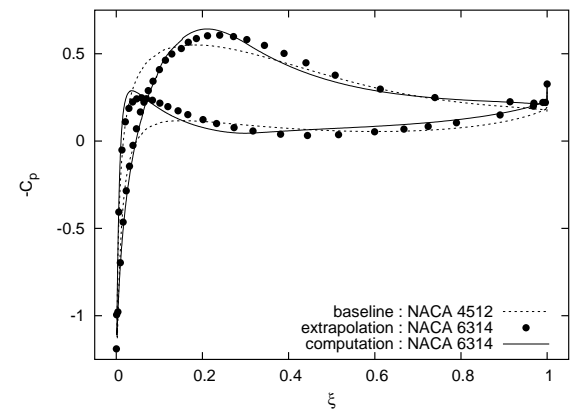

(a) Pressure coefficient

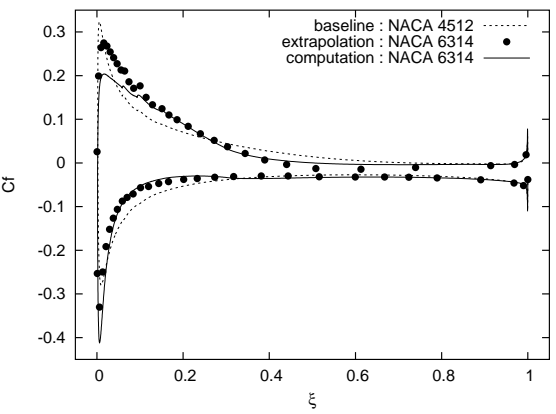

(b) Skin friction coefficient

Figure 11. Nearby solution for the NACA 6314 from the NACA 4512 


\section{Application: gradient-based optimal design}

\subsection{Optimization loop}

Optimal design consists in optimizing (maximizing or minimization) an objective function (or cost function) $\mathcal{J}$ which depends on a vector of design variables a and the flow variables $U(\mathbf{a}, X(\mathbf{a}))$ where $X$ represents the physical domain. The governing equations of the flow $R(\mathbf{a}, U(\mathbf{a}, X(\mathbf{a})))=0$ are considered as constraints which must be satisfied at each step of the design procedure. Some bound constraints must be added to the problem to ensure a realistic solution is found. Thus, the admissible set of the design variables, known as the feasible design space, is usually closed. From a mathematical point of view, the constrained problem may be expressed as:

$$
\begin{array}{ll}
\text { Optimize } & \mathcal{J}(\mathbf{a}, U(\mathbf{a}, X(\mathbf{a}))) \\
\text { Constrained to } & R(\mathbf{a}, U(\mathbf{a}, X(\mathbf{a})))=0 \\
& \mathbf{L}_{\mathbf{i}} \leq \mathbf{a} \leq \mathbf{L}_{\mathbf{s}}
\end{array}
$$

(where $\mathbf{L}_{\mathbf{i}}$ and $\mathbf{L}_{\mathbf{s}}$ may be extended real numbers.) The side constraints can be taken into account inside the optimization algorithm by imposing bounds on the design variables during the globalization step (e.g. when solving the trust-region or line-search sub-problem). The constraint imposed by the flow equations is satisfied at every point within the feasible design space so that it will not explicitly appear in the optimization algorithm presented in the next subsection. Finally, our design optimization loop consists of:

(1) Generation of the initial airfoil shape $X_{0}$

(2) Initialization of the design variables $\mathbf{a}_{0}$

(3) Set $k=0$

(4) Adaptive computation of $U_{k}$ and its sensitivities

(5) Evaluation of the objective function $\mathcal{J}$ and its gradient

(6) Evaluation of $\mathbf{a}_{k+1}$ by the optimization algorithm

(7) Test for convergence, and if necessary increment $k$ and go to step (4)

Step (6) may require additional calls to step (5) as discussed below.

\section{Optimization algorithm}

A gradient-based optimization strategy is chosen to find optimal parameter values. Because of the computational burden of the flow analysis, one must use an optimizer requiring a small number of evaluations of the objective function and its gradient.

Hence, a quasi-Newton algorithm is used for the present study. Considering the maximization of an objective function $\mathcal{J}(\mathbf{a})$, an approximate hessian is initialized with $H_{0}=\mathcal{J}\left(\mathbf{a}_{0}\right) I$ and then updated using a BFGS secant strategy. At the current design point $\mathbf{a}_{k}$, the next values of the parameters are chosen by solving a trust-region sub-problem (Dennis Jr. et al., 1983):

$$
\max _{\left\|\mathbf{s}_{k}\right\| \leq \delta_{k}} \mathcal{J}\left(\mathbf{a}_{k}\right)+\nabla \mathcal{J}\left(\mathbf{a}_{k}\right)^{T} \mathbf{s}_{k}+\frac{1}{2} \mathbf{s}_{k}^{T} H_{k} \mathbf{s}_{k}
$$


with $\mathbf{a}_{k+1}=\mathbf{a}_{k}+\mathbf{s}_{k}$ if the required sufficient decrease conditions are met. This globalization strategy allows convergence for a wider range of initial parameter values. For details on the secant strategy and globalization methods, the reader is referred to Dennis and Schnabel (Dennis Jr. et al., 1983). However, this algorithm requires the evaluation of the objective function $\mathcal{J}$ and its gradient $\nabla \mathcal{J}=\frac{d \mathcal{J}}{d \mathbf{a}}$. The latter is computed using the sensitivities of the flow variables and the sensitivities of geometric quantities as detailed for each specific case in what follows.

\subsection{Drag and lift matching}

This first optimal design problem aims at verifying the correctness of the proposed approach. Starting from an arbitrary choose wing section, we ask the optimization algorithm to find a profile that matches the targeted Drag and Lift coefficients. This is a common problem in aerodynamic design: one must find a wing section generating sufficient lift to sustain flight. Matching target Lift and Drag is also a common subproblem encountered in multi-point design.

The matching problem is defined by an objective function measuring the discrepancy between the current airfoil characteristics $\left(C_{L}, C_{D}\right)$ to their target values:

$$
\mathcal{J}=\frac{\omega_{1}}{2}\left(C_{D}-C_{D}^{*}\right)^{2}+\frac{\omega_{2}}{2}\left(C_{L}-C_{L}^{*}\right)^{2}
$$

where the star coefficients are the target Drag and Lift coefficients. The weight $\omega_{1}$ and $\omega_{2}$ are used to yield a balanced influence to the Drag and Lift independently of their magnitudes:

$$
\omega_{1}=1 \quad ; \quad \omega_{2}=\frac{C_{D}^{*}}{C_{L}^{*}}
$$

Finally, the minimum of this objective function is known to be zero so that the optimal design procedure can be checked because it should find a known global minimum of the cost function.

The design variables are the three shape parameters that define a wing section of the NACA 4-digit series of airfoils: $\mathbf{a}=[e, m, p]^{T}$. The following bounds (for a unit chord) are imposed on the design variables for the optimization process to converge to a feasible design:

$$
e \in[0.1,0.25] \quad ; \quad m \in[0,0.09] \quad ; \quad p \in[0.3,0.7]
$$

That is, the thickness is constrained to be between 10 and $25 \%$ of the chord, the maximum ordinate of the mean line can not exceed $9 \%$ of the chord and its position is forced to lie between 30 and $70 \%$ of the chord, i.e. not too close to the leading and the trailing edge (since it clearly leads to an unrealistic wing section). The angle of incidence can also be taken as a fourth design parameter, and will be studied in the next cases, since it influences the aerodynamic performance of the airfoils (Lépine 
et al., 2001). In the present case, the angle of attack is fixed because its influence dominates that of the other parameters $(e, m$ and $p)$. The airfoil performance is thus determined by the three shape parameters rather than mostly by $\alpha$. This turns out to be a more challenging matching problem. It is worth noting that the optimal solution is not necessarily unique in the feasible design space since several wing sections can have the same Drag and Lift (thus the objective function may have several global minimum). This means that the optimal solution depends on the choice of the initial airfoil and on the initial trust region for obvious reasons. However, this does not cause any limitations because the optimization procedure is set to find one optimal solution (for a given initial point) and not all of them. Finally, one of the main drawbacks of gradient-based algorithms is the possibility that they get stuck in a local minimum (Vanderplaats, 1999). In the present case, we know the global minimum and thus we can monitor whether or not it has been reached by the optimizer. If the global minimum is not reached, the design exercise can always be restarted with a different initial point.

The target values of Drag and Lift are set to typical values for a fixed angle of incidence of $5^{\circ}$ of a NACA airfoil:

$$
C_{D}^{*}=0.14000 \quad ; \quad C_{L}^{*}=0.22000
$$

The initial airfoil is arbitrarily chosen as a NACA 8416 profile. Its Drag and Lift coefficients are:

$$
C_{D}^{0}=0.15833 \quad ; \quad C_{L}^{0}=0.17957
$$

Thus, we ask the optimizer to find a profile that increases the Lift by more than $18 \%$ while reducing Drag by $13 \%$. The optimization process is stopped when the variation of the function value or that of its gradient is outside a confidence interval that has been determined during the grid refinement study (see Section 5.3) or if the variation of all the parameters values become too small (less than $1.10^{-5}$ ).

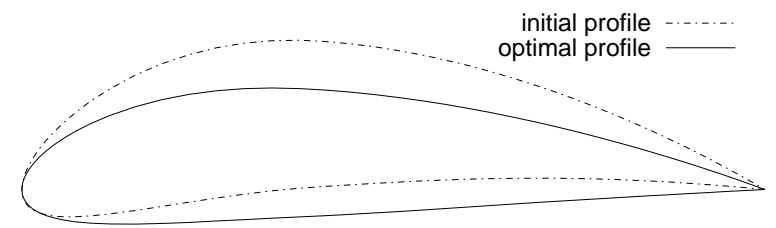

Figure 12. Drag and Lift matching: profiles

Figure 12 shows the initial and optimal profiles of the Drag and Lift matching exercise. Both the camber and the thickness of the initial profile have been reduced to match the Lift and Drag targets. The optimization path is reported in Table 3 which presents the evolution of the design parameters, the value of the objective function and 
of its gradient during the optimization. Figure 13 shows the optimization history of the objective function along with its two contributing terms measuring the performance in terms of Drag and Lift matching respectively. Eleven optimization iterations were necessary to reach the global minimum of the objective function. Both the thickness and the camber have been reduced to reach the targeted Drag and Lift coefficient. Initially, the thickness is reduced until it is in the right range for obtaining the desired performance. The optimizer then works mostly on the maximum camber value and location while refining the thickness parameter. Note that the first two iterates were rejected by the trust-region algorithm indicating that the initial hessian was the wrong scale.

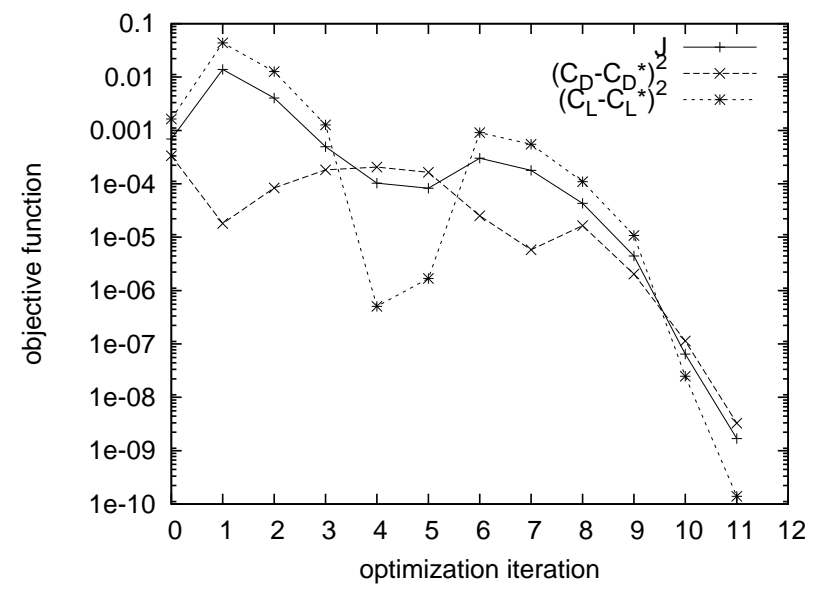

Figure 13. Drag and Lift matching: optimization history

\subsection{Lift-to-Drag ratio maximization}

This section looks at a different design problem: the maximization of the Liftto-Drag ratio for the NACA 4-digit series of profiles. Thus, we ask the optimization procedure to maximize the objective function which is now the Lift-to-Drag ratio:

$$
\mathcal{J}=\frac{C_{L}}{C_{D}}
$$

The design variables are $e, m, p$ and $\alpha$ the angle of incidence. The same bounds as in the previous Section are imposed on the shape variables. The angle of incidence is constrained to lie between 0 and 10 degrees that is $\alpha \in[0,0.175]$ where the angle is expressed in radians. The initial parameter values are set as follows:

$$
e=0.15 \quad ; \quad m=0.05 \quad ; \quad p=0.5 \quad ; \quad \alpha=0
$$


Table 3. Drag and Lift matching: optimization path

\begin{tabular}{lccccrrr}
\hline It. & $e$ & $m$ & $p$ & $\mathcal{J}$ & \multicolumn{1}{c}{$\frac{\partial}{\partial e} \mathcal{J}$} & \multicolumn{1}{c}{$\frac{\partial}{\partial m} \mathcal{J}$} & \multicolumn{1}{c}{$\frac{\partial}{\partial p} \mathcal{J}$} \\
\hline 0 & 0.16 & 0.08 & 0.40 & $0.6910^{-3}$ & $0.8110^{-1}$ & $-0.1510^{-2}$ & $-0.3210^{-2}$ \\
$1^{\star}$ & 0.08 & 0.08 & 0.40 & $0.1410^{-1}$ & $-0.4110^{+0}$ & $0.3410^{-1}$ & $0.1610^{-1}$ \\
$2^{\star}$ & 0.11 & 0.08 & 0.40 & $0.4110^{-2}$ & $-0.2310^{+0}$ & $0.1610^{-1}$ & $0.1210^{-1}$ \\
3 & 0.13 & 0.08 & 0.40 & $0.5010^{-3}$ & $-0.7310^{-1}$ & $0.7610^{-2}$ & $0.4610^{-2}$ \\
4 & 0.14 & 0.08 & 0.40 & $0.1010^{-3}$ & $0.9510^{-3}$ & $0.3710^{-2}$ & $0.7610^{-3}$ \\
5 & 0.14 & 0.07 & 0.40 & $0.8210^{-4}$ & $0.1910^{-2}$ & $0.3710^{-2}$ & $0.5310^{-3}$ \\
6 & 0.14 & 0.01 & 0.38 & $0.3010^{-3}$ & $0.4510^{-1}$ & $-0.1610^{-1}$ & $0.1010^{-3}$ \\
7 & 0.14 & 0.02 & 0.39 & $0.1810^{-3}$ & $0.4010^{-1}$ & $-0.1310^{-1}$ & $-0.1110^{-3}$ \\
8 & 0.14 & 0.05 & 0.39 & $0.4310^{-4}$ & $0.2010^{-1}$ & $-0.3110^{-2}$ & $-0.3610^{-3}$ \\
9 & 0.13 & 0.04 & 0.37 & $0.4510^{-5}$ & $-0.6410^{-2}$ & $0.2310^{-2}$ & $0.9410^{-4}$ \\
10 & 0.13 & 0.04 & 0.37 & $0.6510^{-7}$ & $0.3310^{-3}$ & $-0.2610^{-3}$ & $-0.2510^{-5}$ \\
11 & 0.13 & 0.04 & 0.37 & $0.1710^{-8}$ & $0.1610^{-4}$ & $0.2310^{-4}$ & $-0.7810^{-7}$ \\
\hline \multirow{7}{*}{ steps were rejected and trust-region radius was reduced } \\
\hline
\end{tabular}

As can be seen in Figure 15, 16 iterations were necessary for reaching the optimum. The objective function has been increased by a factor of 15 . However, most of the gain is achieved after only 10 iterations. The final iterations yield marginal improvement in the design. The optimal profile can be compared to the initial shape in Figure 14. Clearly, the thickness has been reduced while the maximum camber has been increased and its position has been moved toward the trailing edge of the wing section. Figure 15 shows the evolution of the objective function, the drag and lift coefficients. As can be seen, the bulk of the gain in the objective function comes from the huge improvement of the lift coefficient during the optimization. Indeed, the lift coefficient increases from 0.03393 for the initial configuration to 0.60708 for the optimal configuration.

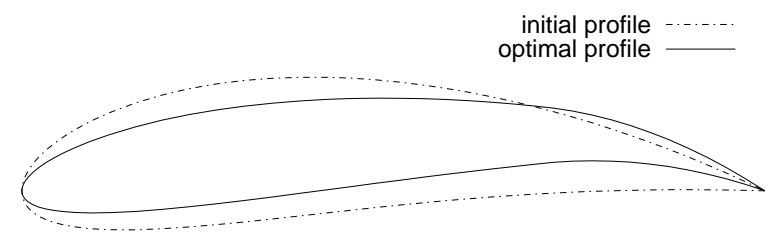

Figure 14. Lift-to-Drag maximization: profiles

This is achieved by initially increasing the angle of incidence up to its maximum bound which leads to a direct enhancement of the lift. Then, the maximum camber position (controlled by the parameter $p$ ) is moved from the middle of the chord to the trailing edge of the profile while the mean line deviation (controlled by the parameter $m$ ) is also increased. Therefore, the camber of the profile has been significantly 
increased as can be easily seen in Figure 14, the deviation of the flow is much higher so that the global force applied by the fluid on the airfoil is also much higher. As one would expect, these geometric modifications also result in an increase of the drag coefficient since a part of it can be directly related to the lift coefficient. Indeed, the drag coefficient increases from 0.13372 for the initial configuration to 0.16209 for the optimal configuration (21\%). However, the increase of the drag coefficient is small compared to that of the lift coefficient so that the magnitude of the objective function is greatly increased during the optimization process and thus the performance of the airfoil is significantly enhanced. Indeed, the lift-induced drag effect is balanced by the fact that the thickness of the profile has been reduced during the optimization process. This change in the parameter $e$ is directly responsible for a reduction of drag. As a consequence, the drag increase has been limited while the lift performance has been greatly improved. It is worth noting that the optimal drag-to-lift ratio is typical of a take-off or landing configuration since the lift is high while the drag is important but limited. The optimal profile looks like a wing section with a plain flap deployed as is the case during landing. The enhanced lift allows the aircraft to fly more slowly and to steepen its approach to the landing site. Thus, the optimal configuration found by the optimization algorithm is physically coherent and realistic.

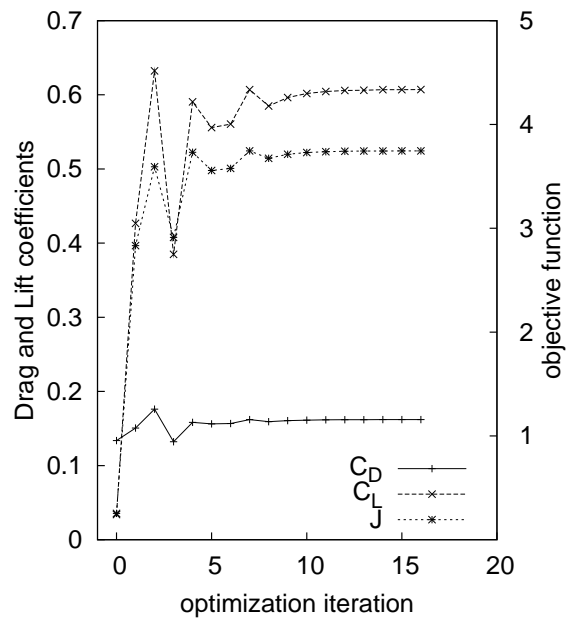

(a) $C_{D}, C_{L}$ and Objective function

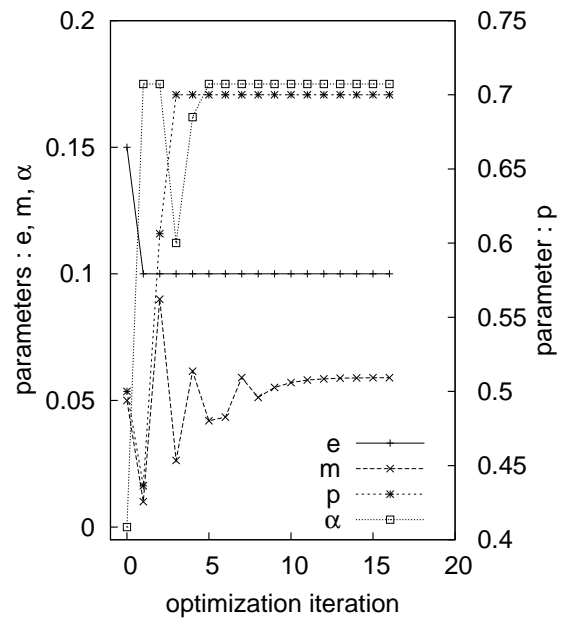

(b) Parameters

Figure 15. Lift-to-Drag maximization: optimization history

\subsection{Drag minimization with constrained Lift}

The last optimization exercise carried out is the Drag minimization of the family of profiles using the four design parameters of the previous Section. A reduction in the Drag will typically result in a reduction of the Lift due to the decrease in the thickness 
and the camber of the airfoil. Thus, for the optimal design to be relevant in a practical context, the Drag minimization has to be performed with a constraint on the Lift. The

$$
\begin{array}{lll}
\text { problem can be expressed as: } & \text { Minimize } & \mathcal{J}_{\mathcal{D}}=C_{D} \\
& \text { Constrained to } & C_{L} \geq C_{L}^{*}
\end{array}
$$

where $C_{L}^{*}$ is the imposed minimum Lift. There are numerous techniques to handle constrained multi-variable functions problem (Vanderplaats, 1999). Here, we use the penalty function method based on a penalized objective function:

$$
\mathcal{J}=\mathcal{J}_{\mathcal{D}}+r \mathcal{H}^{2}
$$

with $r$ the penalty parameter and $\mathcal{H}$ a penalty function that expresses how the inequality-constraint is satisfied. The following choice ensures that no penalty is imposed if the constraint is satisfied:

$$
\mathcal{H}= \begin{cases}C_{L}-C_{L}^{*} & \text { if } C_{L} \geq C_{L}^{*} \\ 0 & \text { otherwise }\end{cases}
$$

A trade-off has to be made when choosing the value of the penalty parameter so that the minimization problem will not yield constraint violations that are too large and problems that are too poorly conditioned from a numerical standpoint. We set $r$ to 100 based on previous experience. The minimum allowable Lift coefficient is set to $C_{L}^{*}=0.2$ for the minimization problem to be of practical interest.

As in the previous Section, the initial profile is chosen to be a NACA 8416 at $5^{\circ}$ of incidence. Note than this profile does not satisfy the constraint on the Lift (see Section 7.2). The minimum of the penalized objective function is reached in 18 iterations. The optimal profile fully satisfies the Lift constraint and the Drag has been reduced by 24\%. The initial and optimal profiles are presented in Figure 16.

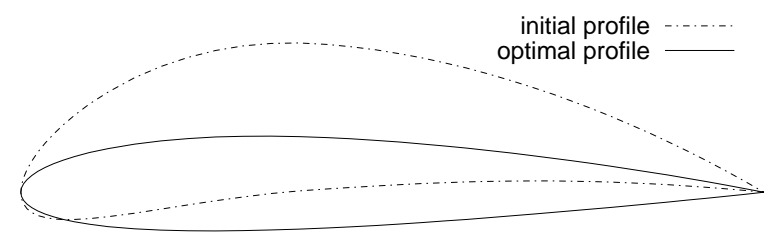

Figure 16. Drag minimization: profiles

The optimization path is summarized in Figure 17. The thickness parameter and the maximum camber parameter have been decreased to their respective lower bound while the angle of attack is adjusted to satisfy the lift constraint. The sharp peak of the objective function is due to the penalty term as the constraint is violated during the optimization process. It is worth noting the Lift of the optimal profile found is the minimum allowable Lift chosen. This was expected because no bonus is associated with a Lift greater that $C_{L}^{*}$. Since a change in the parameters has most of the time opposite effects on the Drag and Lift coefficients, the Lift constrained minimization of the Drag is obtained for a profile that matches the minimum allowable Lift. 


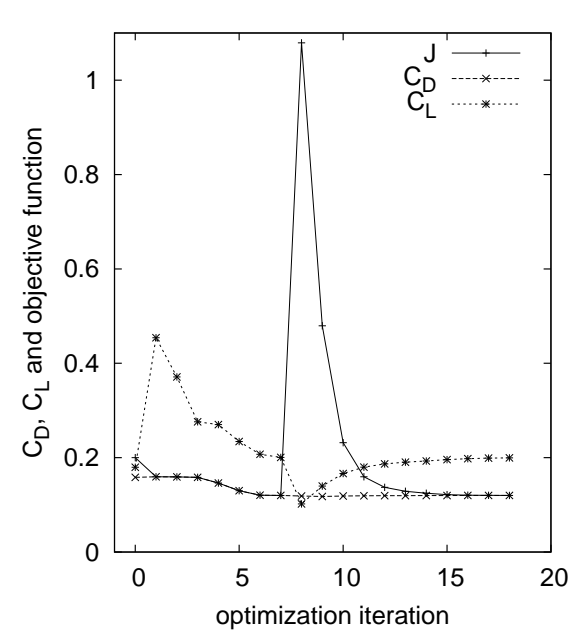

(a) $C_{D}, C_{L}$ and Objective function

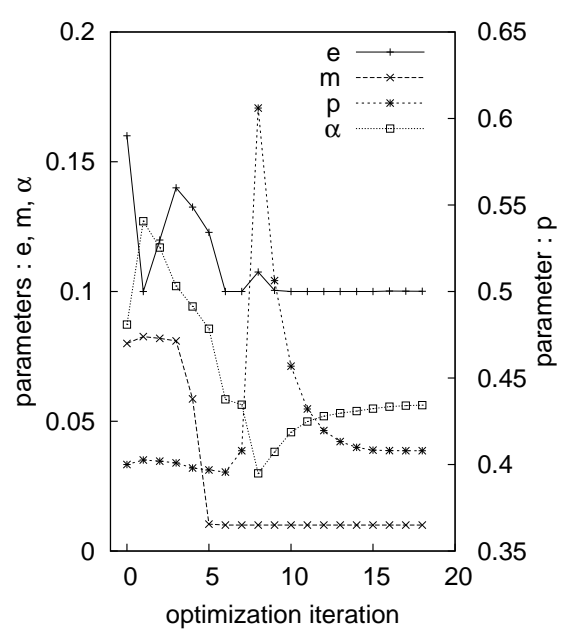

(b) Parameters

Figure 17. Drag minimization: optimization history

\section{Summary and concluding remarks}

A continuous shape sensitivity equations method has been presented. Details have been given on the suitable methodology to handle parameter dependent boundaries: a proper description of the computational boundary, the necessary use of the Lagrangian sensitivities and the correct derivation of transpiration terms for computing the derivatives of physical quantities. To illustrate the correctness of the proposed approach, numerical applications have been considered based on flows around the NACA 4-digit series family of wing sections. All flow and sensitivity equations are solved using an adaptive finite element method driven by a posteriori estimates of the error on the variables. A grid convergence study has been carried out to characterize the suitable mesh density and to determine its influence on the outputs.

The first application of the methodology shows how sensitivity information can be used in a non optimization problem: the fast evaluation of flows on nearby geometries. This is achieved by using Taylor series in parameter space. This methodology has been pushed to its limits by considering large changes in the airfoil geometries that correspond to large modification of the shape parameter values. Even in such an extreme situation, the extrapolated solution yields useful information. The second application concerns the use of the Sensitivity Equations Method for gradient-based optimal design. A BFGS optimization algorithm is used to find the optimal values of shape parameters. Flow and sensitivity information are employed to calculate the values and gradients of the design objective function. Three different optimization problems were considered to assess the validity of the proposed approach. In this con- 
text, further work will aim at developing a richer design space that the one offered by the NACA four-digit series family of airfoils.

Acknowledgments

This work was supported in part by the Canada Research Chair Program and by the National Science and Engineering Research Council of Canada (NSERC, Government of Canada).

\section{References}

A.Griewank, Evaluating Derivatives, SIAM, Philadelphia, PA, 2000.

Anderson W. K., Newman J. C., Whitfield D. L., Nielsen E. J., " Sensitivity Analysis of NavierStokes Equations on Unstructured Meshes unsing Complex Variables", AIAA Journal, vol. 39, n ${ }^{\circ} 1$, p. 56-53, 2001.

Blackwell B. F., Dowding K. J., Cochran R. J., Dobranich D., “Utilization of sensitivity coefficients to guide the design of a Thermal battery", Proceedings of the 1998 ASME/IMECE, ASME, Anaheim, CA, p. 73-82, 1998. HTD-Vol. 561-5.

Borggaard J., Burns J., “ A PDE sensitivity equation method for optimal aerodynamic design”, J. Comput. phys., vol. 136, n $^{\circ}$ 2, p. 367-384, 1997.

Borggaard J., Pelletier D., “ Optimal Shape Design in Forced Convection Using Adaptive Finite Elements”, 36th AIAA Aerospace Sciences Meeting and Exhibit, Reno, NV, January, 1998. AIAA Paper 98-0908.

Borggaard J., Pelletier D., Vugrin K., “ On Sensitivity Analysis for Problems with Numerical Noise", 9th AIAA/ISSMO Symposium on Multidisciplinary Analysis and Optimization, Atlanta, GA, September, 2002. AIAA Paper 2002-5553.

Dennis Jr. J., Schnabel R., Numerical Methods for Unconstrained Optimization and Nonlinear Equations, Prentice-Hall, 1983.

Duvigneau R., Pelletier D., “ Evaluation of Nearby Flows by a Shape Sensitivity Equation Method", 43th AIAA Aerospace Sciences Meeting and Exhibit, Reno, NV, Jan., 2005 a. AIAA Paper 2005-0127.

Duvigneau R., Pelletier D., “ On Accurate Boundary Conditions for a Shape Sensitivity Equation Method", International Journal for Numerical Methods in Fluids, vol. 50, p. 1417-164, 2005b.

Godfrey A. G., Cliff E. M., “ Direct Calculation of Aerodynamic Force Derivatives: A Sensitivity-Equation Approach", 36th AIAA Aerospace Sciences Meeting and Exhibit, Reno, NV, January, 1998. AIAA Paper 98-0393.

Godfrey A. G., Cliff E. M., “Sensitivity Equations for Turbulent Flows”, 39th AIAA Aerospace Sciences Meeting and Exhibit, Reno, NV, Jan., 2001. AIAA Paper 2001-1060.

Gunzburger M. D., Perspectives in Flow Control and Optimization, SIAM, 2002.

Haug E. J., Choi K., Komkov V., Design sensitivity analysis of structural systems, vol. 177 of Mathematics in science and engineering, Academic Press, Orlando, 1986. 
60 REMN - 17/2008. Shape design in aerodynamics

Hay A., Etienne S., Duvigneau R., Pelletier D., “Evaluation of Flows on Nearby Geometries by a Shape Sensitivity Equation Method", 44th AIAA Aerospace Sciences Meeting and Exhibit, Reno, NV, 9-12 January, 2006. AIAA Paper 2006-1296.

Ilinca F., Pelletier D., “ A Continuous shape sensitivity equation method for unsteady laminar flows", European Conference on Computational Fluid Dynamics, ECCOMAS CFD 2006, TU Delft, The Netherlands, september, 2006.

Ilinca F., Pelletier D., Borggaard J., “ A Continuous Second Order Sensitivity Equation Method for Time-Dependent Incompressible Laminar Flows”, 35th AIAA Fluid Dynamics Conference and Exhibit, Toronto, ON, June, 2005. AIAA Paper 2005-5252.

Kleiber M., Antúnez H., Hien T. D., Kowalczyk P., Parameter Sensitivity in Nonlinear Mechanics, John Wiley and Sons, 1997.

Lépine J., Guibault J., Trépanier J.-Y., Pépin F., “ Optimized NonUniform Rational B-Spline Geometrical Representation for Aerodynamic Design of Wings", AIAA Journal, vol. 39, $\mathrm{n}^{\circ}$ 11, p. 2033-2041, 2001.

Limache A., Aerodynamic Modeling Using Computational Fluid Dynamics and Sensitivity Equations, PhD thesis, Virginia Polytechnic Institute and State University, Blacksburg, VA, 2000.

Lu S.-Y., Sagaut P., “ Direct sensitivity analysis for smooth unsteady compressible flows using complex differentiation Design”, International Journal for Numerical Methods in Fluids, 2006.

Lyness J., Moler C., “ Numerical differentiation of analytic functions”, SIAM J. Numer. Anal, vol. $4, n^{\circ} 2$, p. 202-210, June, 1967.

Mahieu J., Etienne S., Pelletier D., Borggaard J., “ A Second-order sensitivity equation method for laminar flow”, International Journal of Computational Fluid Dynamics, vol. 19, $\mathrm{n}^{\circ} 2$, p. 143-157, February, 2005.

Martins J. R. R. A., Stradza P., Alonso J. J., “ The complex-step derivative approximation”, ACM transactions on mathematical software - TOMS, vol. 29, $\mathrm{n}^{\circ}$ 3, p. 245-262, 2003.

Pelletier D., " Adaptive Finite Element Computations of Complex Flows", International Journal for Numerical Methods in Fluids, vol. 31, p. 189-202, 1999.

Pelletier D., Ilinca F., “ Adaptive Remeshing for the $k-\epsilon$ Model of Turbulence”, AIAA Journal, vol. 35, n 4, p. 640-646, 1997.

Pelletier D., Roache P. J., Verification and Validation in Computational Heat Transfer, Handbook of Numerical Heat Transfer, 2nd edn, Wiley, Hoboken, New Jersey, chapter 13, March, 2006.

Peraire J., Vahdati M., Morgan K., Ziekiewicz O., “ Adaptive Remeshing for Compressible Flow Computations", Journal of Computational Physics, vol. 72, n 2, p. 449-466, 1987.

Putko M., Newman P., Taylor A., Green L., “ Approach for uncertainty propagation and robust design in CFD using sensitivity derivatives", 15th AIAA Computational Fluid Dynamics Conference, Anaheim, CA, June, 2001. AIAA Paper 2001-2528.

Roache P. J., Verification and Validation in Computational Science and Engineering, Hermosa publishers, Albuquerque, NM, 1998.

Sherman L. L., Taylor III A. C., Green L., Newman P. A., Hou G. W., Korivi V. M., “ First- and second-order aerodynamic sensitivity derivatives via automatic differentiation", Journal of Computational Physics, vol. 129, n 2, p. 307-331, 1996. 
Stanley L. G., Stewart D. L., Design Sensitivity Analysis: Computational Issues of Sensitivity Equation Methods, vol. 25 of Frontiers in Applied Mathematics, SIAM, Philadelphia, 2001.

Turgeon E., Pelletier D., " Verification and Validation in CFD Using an Adaptive Finite-Element Method”, CASI Journal, vol. 48, n 4, p. 219-231, December, 2002.

Turgeon É., Pelletier D., Borggaard J., “ A Continuous Sensitivity Equation Approach to Optimal Design in Mixed Convection”, 33rd AIAA Thermophysics Conference, Norfolk, VA, Jun.-Jul., 1999. AIAA Paper 99-3625.

Turgeon É., Pelletier D., Borggaard J., “ A Continuous Sensitivity Equation Method for Flows with Temperature Dependent Properties", 8th AIAA/NASA/USAF/ISSMO Symposium on Multidisciplinary Analysis and Optimization, Long Beach, CA, Sep., 2000a. AIAA Paper 2000-4821.

Turgeon É., Pelletier D., Borggaard J., “ A General Continuous Sensitivity Equation Formulation for Complex Flows", 8th AIAA/NASA/USAF/ISSMO Symposium on Multidisciplinary Analysis and Optimization, Long Beach, CA, Sep., 2000b. AIAA Paper 2000-4732.

Turgeon É., Pelletier D., Borggaard J., “ A General Purpose Sensitivity Equation Formulation for Complex Flows", Proceedings of the 8th Annual Conference of the Computational Fluid Dynamics Society of Canada, vol. 2, Montréal, Canada, p. 697-704, June 11-13, 2000c.

Turgeon É., Pelletier D., Borggaard J., “ Application of a Sensitivity Equation Method to the $k-$ $\epsilon$ Model of Turbulence", 15th AIAA Computational Fluid Dynamics Conference, Anaheim, CA, Jun., 2001a. AIAA Paper 2001-2534.

Turgeon É., Pelletier D., Borggaard J., “ Computation of Airfoil Flow Derivatives Using a Continuous Sensitivity Equation Method", 8th CASI Aerodynamics Symposium, Toronto, Canada, April, 2001b.

Turgeon É., Pelletier D., Borggaard J., “ A General Continuous Sensitivity Equation Formulation for the $k-\epsilon$ Model of Turbulence", 31st AIAA Fluid Dynamics Conferenceand Exhibit, Anaheim, CA, Jun., 2001c. AIAA Paper 2001-3000.

Turgeon É., Pelletier D., Borggaard J., " Sensitivity and Uncertainty Analysis for Variable Property Flows", 39th AIAA Aerospace Sciences Meeting and Exhibit, Reno, NV, Jan., 2001d. AIAA Paper 2001-0139.

Vanderplaats G. N., Numerical optimization techniques for engineering design, third edn, Vanderplaats Research and Development, Colorado Springs, CO, 1999.

Zienkiewicz O. C., Zhu J. Z., “ The Superconvergent Patch Recovery and a Posteriori Error Estimates. Part 1: The Recovery Technique", International Journal for Numerical Methods in Engineering, vol. 33, p. 1331-1364, 1992a.

Zienkiewicz O. C., Zhu J. Z., “ The Superconvergent Patch Recovery and a Posteriori Error Estimates. Part 2: Error Estimates and Adaptivity", International Journal for Numerical Methods in Engineering, vol. 33, p. 1365-1382, 1992 b. 
